\title{
A review of cognitive conflicts research: a meta-analytic study of prevalence and relation to symptoms
}

This article was published in the following Dove Press journal:

Neuropsychiatric Disease and Treatment

4 December 2015

Number of times this article has been viewed

\author{
Adrián Montesano' \\ María Angeles López- \\ González ${ }^{2}$ \\ Luis Angel Saúl ${ }^{2}$ \\ Guillem Feixas' \\ 'Department of Personality, \\ Assessment and Psychological \\ Treatments, University of Barcelona, \\ Barcelona, ${ }^{2}$ Department of Personality, \\ Assessment and Psychological \\ Treatments, Faculty of Psychology, \\ National Distance Education \\ University, Madrid, Spain
}

Correspondence: Adrián Montesano Department of Personality, Assessment and Psychological Treatments, University of Barcelona, Passeig Vall d'Hebron,

171, 08035 Barcelona, Spain

Tel +34 933125023

Email adrianmontesano@gmail.com
Abstract: Recent research has highlighted the role of implicative dilemmas in a variety of clinical conditions. These dilemmas are a type of cognitive conflict, in which different aspects of the self are countered in such a way that a desired change in a personal dimension (eg, symptom improvement) may be hindered by the need of personal coherence in another dimension. The aim of this study was to summarize, using a meta-analytical approach, the evidence relating to the presence and the level of this conflict, as well as its relationship with well-being, in various clinical samples. A systematic review using multiple electronic databases found that out of 37 articles assessed for eligibility, nine fulfilled the inclusion criteria for meta-analysis. Random effects model was applied when computing mean effect sizes and testing for heterogeneity level. Statistically significant associations were observed between the clinical status and the presence of dilemmas, as well as level of conflict across several clinical conditions. Likewise, the level of conflict was associated with symptom severity. Results highlighted the clinical relevance and the transdiagnostic nature of implicative dilemmas.

Keywords: implicative dilemmas, cognitive conflicts, intrapersonal conflicts, meta-analysis

\section{Introduction}

Theorists and researchers from different approaches in psychology and psychotherapy have used the concept of intrapsychic conflict to understand and explain human behavior and psychopathology. Despite its seemingly important role, the relationship between inner conflicts and well-being has so far been an under-researched topic within clinical psychology and many aspects of their etiopathological and motivational implications remain unclear. This scarcity of empirical research may be related to the elusive nature of intrapersonal conflicts and the challenge of developing a reliable tool, which can assess them. There are, however, some empirical approaches worth mentioning. For instance, research on goal systems in personality functioning showed that goal conflicts can influence the level of well-being and life satisfaction, ${ }^{1}$ as well as the level of engagement of patients in therapy. ${ }^{2}$ There are other empirical procedures for the measurement of conflict such as the work of Grosse Holtforth and Grawe ${ }^{3}$ on goals incongruence, Cierpka et $\mathrm{al}^{4}$ on Axis III of their diagnostic system for conflicts, Arnow et $\mathrm{al}^{5}$ on reactance, and Feixas et $\mathrm{al},{ }^{6}$ who have provided some systematic procedures for the measurement of cognitive conflicts.

Concretely, Feixas and Saúl ${ }^{7}$ launched in 1999 the Multi-Center Dilemma Project (www.usal.es/tcp), a multicenter collaborative research program aimed at exploring the role of implicative dilemmas (IDs) in the onset and maintenance of health problems. IDs are a type of cognitive conflict that refers to conflicts among different aspects of 
the self: a dimension in which change desired (eg, symptom improvement) is hindered by the need to maintain personal coherence. During the last decade, the relevance of IDs for different mental and physical health problems has been investigated. Thus, in this article, we first outline the concept of ID and then systematically review the existing empirical research about IDs across a variety of clinical conditions using a meta-analytic methodology.

\section{What is an implicative dilemma?}

The notion of ID is grounded in Kelly's personal construct theory. ${ }^{8}$ The personal construct theory proposed that human beings need to make sense of their experiences to make the world more understandable and predictable. This process is driven by personal meanings, which are made up of personal constructs. These are bipolar in nature (eg, a good person vs a bad person), and are hierarchically organized within the cognitive system. So, individuals may develop a dynamic network of personal meanings that encompass a limited set of personal constructs with different levels of centrality. The most central or core constructs are those that define the person's identity, underpinning stable patterns of behavior, whereas there are more peripheral constructs that, although subordinate to the core, are actively involved in construing events and action tendencies. As the sense of identity lies at the core of the system, changes that imply invalidation of this set of constructs are strongly resisted in order to keep a sense of personal coherence.

An ID is a conflict in which a desired change on a self-appraisal implies, in turn, an unwanted change on a central aspect of one's identity. Such a conflict between a desired self (eg, being calm) and a feared self (eg, becoming selfish) corresponds to a particular arrangement between two types of personal constructs (Figure 1):

1. Discrepant construct are those in which the person perceives a discrepancy between the actual and the ideal self. These are perceived negative attributes that typically signify areas of malaise, such as psychological symptoms, and therefore encourage the person to pursue a change in his/her actual self. In our example, the patient's view of herself is that she is "nervous", whereas her ideal self (and therefore her desired change) is to be "calmer" (Figure 1).

2. Congruent construct are those in which the person perceives similarity between the actual and the ideal self. These are often core constructs that are likely to be connected to personal values, beliefs, and attitudes that define the individual's identity. Therefore, continuity is sought in order to keep a coherent sense of self. The patient mentioned in Figure 1 considers herself as being a "good person" and does not want to become "selfish".

We can identify an ID whenever there exists a significant correlation between the desired pole of the discrepant construct and the unwished pole of the congruent construct within the context of an individual self-system. In other words, the need for change toward achieving the characteristics of the ideal self is countered by the need for personal coherence, which responds somehow in opposition to this movement. For instance, for the patient mentioned in Figure 1, the idea of

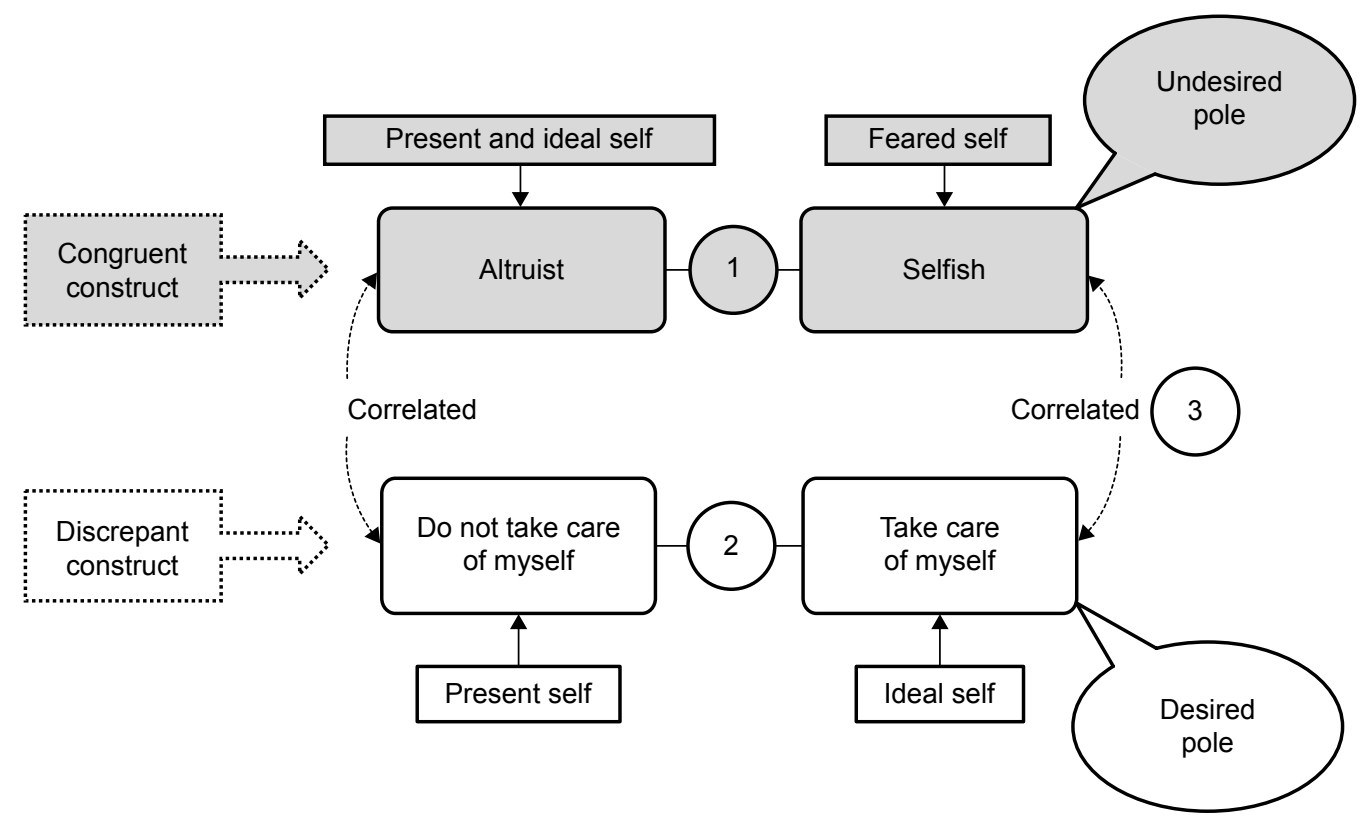

Figure I Example of an implicative dilemma and its components.

Notes: I, congruent construct; 2 , discrepant construct; 3, correlation between constructs. 
attaining her desired self (being "calm") activated her feared self (becoming "selfish"), resulting in her refraining from taking action and therefore in symptom maintenance.

Thus, the central hypothesis of this approach asserts that symptoms are invested with personal meanings (constructs, goals) often involving the core characteristics of the person's own identity. Hence, change may be hindered unless these dilemmas are dealt with in the context of therapy. Conversely, dealing with these conflicts may reduce ambivalence and facilitate readiness to change. ${ }^{9}$

Results from the Multi-Center Dilemma Project suggest that, within clinical samples, there is a higher prevalence of conflicts and individual frequency of conflict (number of IDs within a subject). In addition, the relationship between the levels of conflict and symptom severity has been investigated throughout different targeted samples. However, to our knowledge, these findings have neither been gathered together and synthesized nor analyzed from a global perspective. This article aims to systematically compile the various findings comparing clinical conditions to controls and provide overall summaries of effects sizes through meta-analyses. In doing so, we analyzed three different outcomes. The first two outcomes were investigated using between-group comparisons: 1) the prevalence of participants presenting with IDs; and 2) the participants' level of conflict, understood as the number of IDs within an individual's cognitive system. We then examined the relationship between the level of conflict and symptom severity within the clinical samples.

\section{Methods}

\section{Search procedures}

In order to select the studies that could fulfill the selection criteria, we adopted both formal and informal procedures. ${ }^{10,11}$ The electronic search for articles was carried out using the search equation ["Repertory Grid" OR "Rep Grid”] AND ["Cognitive conflict*" OR "Implicative dilemma*"] in title, abstract or key words, or default settings in non-configurable databases. No limits were set for time, language, or document type. As shown in Figure 2, several databases and specialized journals until December 2014 were searched. The consulted databases were classified as thematic (PsycInfo, EMBASE, and Psicodoc) or multidisciplinary (Academic Search Premier, CSIC-ISOC, DIALNET, E-journals, Scopus, and Web of Science). The journal of Personal Construct Theory and Practice was manually examined as it was not indexed in any database. In addition, leading researchers in the field were contacted in an attempt to recover unpublished articles or articles in press. From the initial 37 independent references retrieved by the search, nine studies fulfilled the selection criteria, and were included in our study (Figure 2). With the aim of verifying the quality of the selected studies, a codebook based on the Newcastle-Ottawa Scale ${ }^{12}$ was developed. The methodological characteristics were coded as follows: 1) definition of inclusion and exclusion criteria; 2) description of the evaluation process; 3) information of the flow of participants (eligible, experimental mortality); 4) assessment of the presence of comorbidity; and 5) control of subjects under psychological or pharmacological treatment. The participants' characteristics coded from the samples of each study were: 1) the mean age; 2) the percentage of females; and 3 ) the type of clinical condition of the participants.

Each study was analyzed independently by two researchers (AM and LÁS) who used the codebook. An overall estimation of the quality of the research was made within a 7-point Likert scale ranging from minimum to maximum. Coders resolved disagreements by consensus, and reached $100 \%$ agreement regarding the studies that would be included in the current investigation. The extraction of data for computing the effect sizes was also performed independently by the two researchers.

\section{Selection criteria of the studies}

Several predefined inclusion criteria were established for this study. First, studies were required to investigate the role of IDs in clinical groups in an empirical manner. Targeted samples could include any kind of psychological disorder or physical health condition. Theoretical papers and case studies were excluded. Second, studies were required to select participants on the basis of a diagnostic system (eg, Diagnostic and Statistical Manual for Mental Disorders) to confirm the clinical status of participants. Third, studies were required to include a control group for statistical comparison. The existence of a control group reduces threats to internal validity and allows the computation of standardized effect sizes. Fourth, only studies that reported the data required to calculate effect sizes were included. Therefore, the frequency of participants presenting with IDs (outcome 1), means and standard deviations of the levels of conflicts (outcome 2), and the correlation with symptom severity (outcome 3 ) needed to be present in the manuscript or available upon request from the authors. Taking into account the authors' linguistic limitations, the studies were required to be written in English, Spanish, Italian, or Portuguese.

\section{Computation of effect sizes}

All the nine studies that were selected used the repertory grid and the software GRIDCOR (version 4.0; Centro de Terapia 


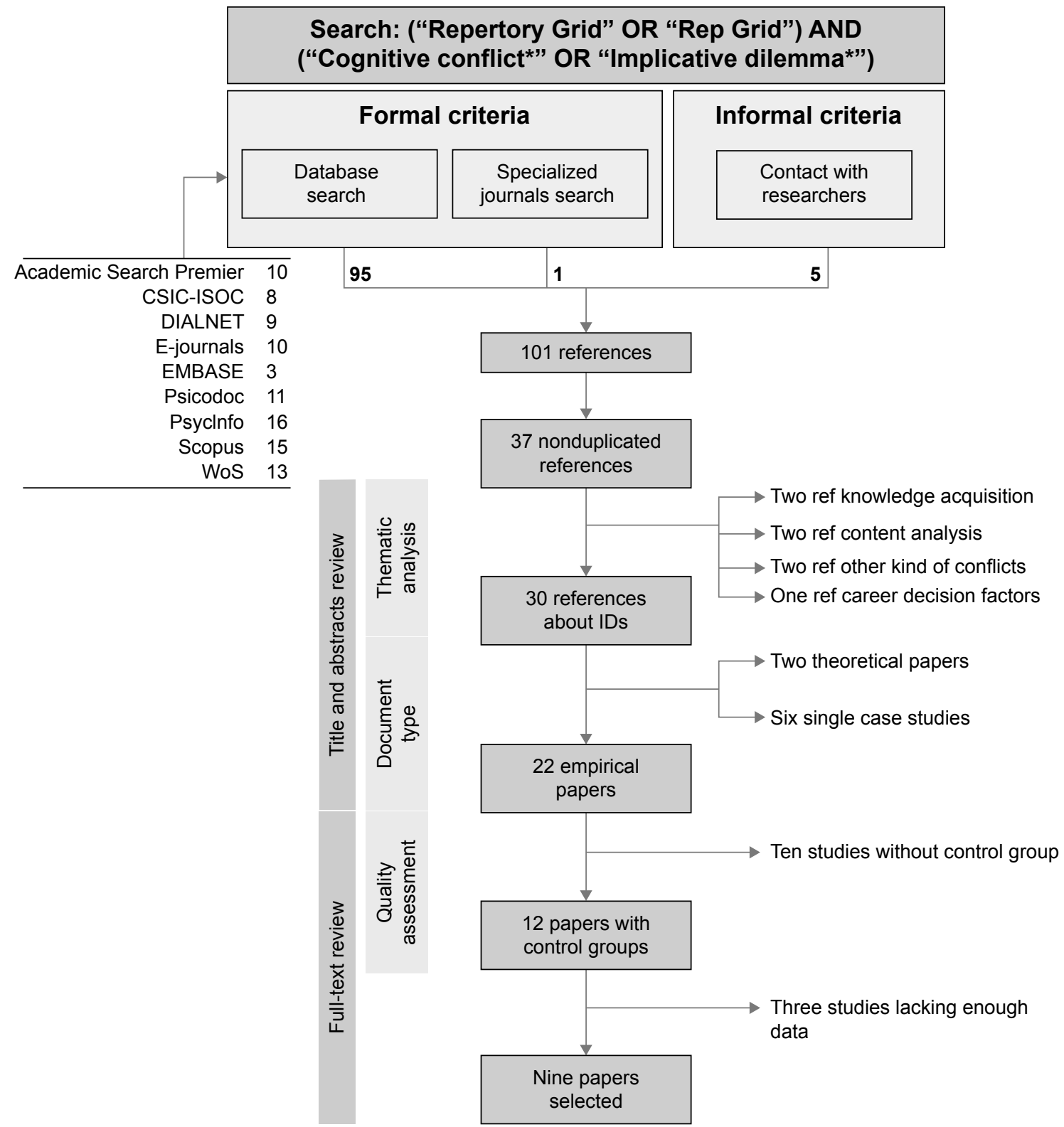

Figure 2 Flowchart of search and selection of studies.

Abbreviations: IDs, implicative dilemmas; ref, reference; WoS, Web of Science.

Cognitiva, Barcelona, Spain $)^{13}$ for detecting IDs. A different type of effect size was calculated for each of the three outcome measures, due to the differences in the nature of the dependent variables. First, the odds ratio (OR) based on a $2 \times 2$ contingency table was used as a measure of association between group membership and presence/absence of IDs; both categorical and dichotomous variables. For calculating $\mathrm{OR}$, we used the following formula:

$$
\mathrm{OR}=\frac{\text { Controls with IDs/clinical with IDs }}{\text { Control without IDs/clinical without IDs }}
$$

Second, the level of conflict was measured in the original studies by counting the number of IDs within a subject with a correction factor for the total number of constructs within the subject's grid (since the number of constructs provided by each participant varies, those providing more personal constructs have a higher chance to present with IDs). As a continuous variable, the standardized mean difference $(d)$ was used to determine the effect size for the level of conflict. For computing $d$, the difference between the mean of the clinical $\left(M_{1}\right)$ and the control groups $\left(M_{2}\right)$ was divided by a pooled estimate of the within-study standard deviation. Thus, 
$d=M_{1}-M_{2} / \sigma$ pooled, where $\sigma$ pooled $=\sqrt{ }\left[\left(\left(\mathrm{n}_{1}-1\right) S_{1}^{2}+\left(\mathrm{n}_{2}-1\right) S_{2}^{2}\right) /\right.$ $\left.\mathrm{n}_{1}+\mathrm{n}_{2}-2\right]$. Finally, correlation coefficients $(r)$ were chosen as effect sizes for the relationship between the level of conflict and symptom severity, as this relationship was expressed in correlational terms within the primary studies. Since the sampling distribution of Pearson's $r$ is not normally distributed, it was converted to Fisher's $z^{11}$ and the confidence interval (CI) was computed using Fisher's $z$. The values of Fisher's $z$ in the CI were then converted back to Pearson's $r$. Cohen' ${ }^{14}$ criteria were used for interpreting the magnitudes of effect sizes.

\section{Statistical analysis}

In order to explore the three outcome measures, separate meta-analyses were carried out for each type of effect size calculated, as well as for the global mean effect. A random effects model was considered the most appropriate model for the current study due to several theoretical reasons, such as the assumption of variable population parameters with regard to IDs, our intention to generalize beyond the included studies, and the smaller risk of applying random effects models to fixed effect data than the opposite. However, for transparency and in accordance with recent standards of meta-analysis procedures, ${ }^{15,16}$ results from both models were reported. The process of analyses consisted of computing the mean effect size with its $95 \% \mathrm{CI}$, the heterogeneity test, $Q$, and the $I$ index to assess the degree of heterogeneity of the mean effect sizes. ${ }^{15}$ Finally, although we searched for and included unpublished articles, a test for publication bias was conducted. ${ }^{17}$ In addition, Egger's test was used to assess funnel plots for significant asymmetry. All the statistical analyses were carried out using the software Comprehensive Meta-Analysis ${ }^{18}$ (version 2.2.064; Biostat, Inc, Englewood, NJ, USA).

\section{Results}

\section{Included studies}

Overall, the study consisted of 27 outputs based on three outcome measures of the nine studies that were finally selected.
The total sample size consisted of 2,630 individuals, 774 in the clinical groups and 1,856 in the controls (median sample size $=292$ ). The two researchers conducting the searches and the quality assessment concurred to a great extent on both the selection of studies $(\kappa=1)$ and their classification (intraclass correlation of 0.89). Standard diagnostic clinical interviews conforming to Diagnostic and Statistical Manual for Mental Disorders- $I V^{19}$ were used to identify participants in the six studies concerning psychological disorders (major depression, dysthymia, bulimia, anxiety, and a sample of mixed disorders). Clinical diagnoses were used in the two studies, which analyzed functional health problems (fibromyalgia and irritable bowel syndrome). The study of Soldevilla et $\mathrm{al}^{20}$ concerning intimate partner violence also used a specific clinical interview. Since the same instrument and procedure were used to identify IDs in all of the studies, there was high homogeneity with regard to the evaluation process. However, only a few studies reported specific information in relation to the flow of participants and comorbidity. In addition, no study controlled for the influence of psychological or pharmacological treatment. A review of the instruments applied in the different studies to assess symptom severity revealed that the most frequently used instrument was the Symptom Checklist $90-R,{ }^{21}$ which was used in five studies. Two studies used the general symptom index, ${ }^{6,22}$ another two used the anxiety subscale, ${ }^{20}$ and one study used the depression subscale. ${ }^{23}$ Three studies ${ }^{23-25}$ used the Beck Depression Inventory II Manual, second edition ${ }^{26}$ and only one study ${ }^{27}$ used the State-Trait Anxiety Inventory: Bibliography. ${ }^{28}$

\section{Effect sizes}

For each outcome measure, we carried out a meta-analysis to obtain an estimate of the mean effect size and then tested for heterogeneity. The effect sizes and related statistics are presented in Table 1. As shown in this table, the global result for the 27 outcomes gave a statistically significant mean effect size $\left(d_{+}=0.66 ; 95 \%\right.$ CI: $\left.0.53-0.78\right)$, which, according to Cohen's ${ }^{14}$ classification, implies a medium-to-large

Table I Summary results for the effects sizes as a function of the outcome measure

\begin{tabular}{|c|c|c|c|c|c|c|c|c|}
\hline Outcome & Model & $k$ & Type of effect & Effect size & $95 \% \mathrm{CI}$ & Q & $P$-value & $I^{2}$ \\
\hline \multirow[t]{2}{*}{ Prevalence } & Fixed & 9 & OR & 3.013 & $2.46 \mathrm{I}-3.689$ & 15.327 & 0.053 & 47.806 \\
\hline & Random & & & 3.426 & $2.505-4.686$ & & & \\
\hline \multirow[t]{2}{*}{ Level of conflict } & Fixed & 9 & $d$ & 0.597 & $0.499-0.695$ & 9.959 & 0.268 & 19.672 \\
\hline & Random & & & 0.606 & $0.489-0.722$ & & & \\
\hline \multirow[t]{2}{*}{ Symptom severity } & Fixed & 9 & $r$ & 0.352 & $0.287-0.413$ & $5.04 I$ & 0.753 & $<0.001$ \\
\hline & Random & & & 0.352 & $0.287-0.413$ & & & \\
\hline \multirow[t]{2}{*}{ Global results } & Fixed & 27 & $d$ & 0.658 & $0.532-0.783$ & 4.542 & 0.805 & $<0.001$ \\
\hline & Random & & & 0.658 & $0.532-0.783$ & & & \\
\hline
\end{tabular}

Abbreviations: OR, odds ratio; $k$, number of studies; $\mathrm{Cl}$, confidence interval; $Q$, heterogeneity statistic; $I^{2}$, heterogeneity index. 


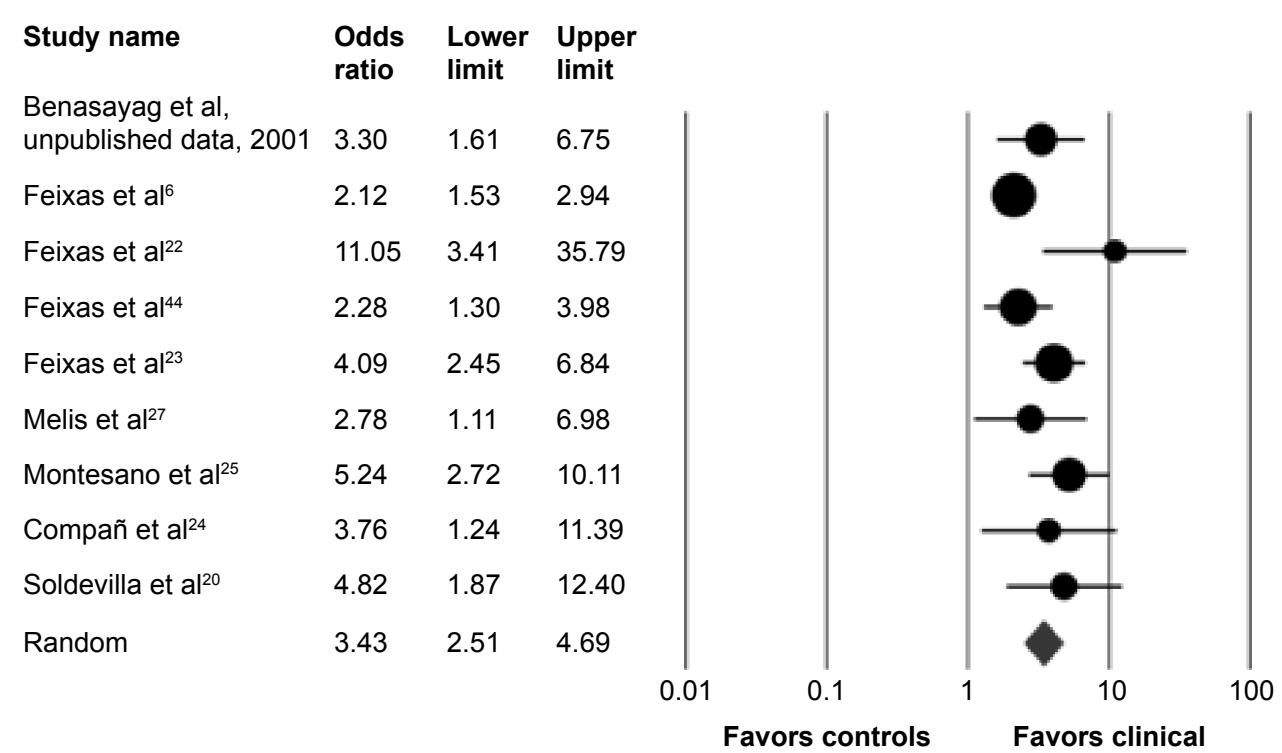

Figure 3 Forest plot for meta-analysis of the prevalence of subjects presenting with implicative dilemmas.

magnitude of differences between targeted samples and controls with regard to IDs.

In all of the meta-analyses reported in Table 1, the heterogeneity between the individual effect sizes of the studies was low. We did not only interpret the fact that all the $Q$ values were not significant but also inspected the heterogeneity index, $I^{2}$, given that the number of studies included was relatively small. As shown, this index remained below $50 \%$ for all of the performed analyses. The absence of significant heterogeneity precluded the examination of moderators accounting for differences between effect sizes across the different studies.

Regarding the prevalence of subjects who presented with IDs, a medium effect size was found (OR $=3.43 ; 95 \% \mathrm{CI}$ : 2.50-4.69), indicating that clinical participants were almost
3.5 times more likely to present at least one ID. All the ORs we found were above 1, indicating the higher prevalence of IDs in clinical samples. Although below the threshold, $I^{2}$ yielded a high level of heterogeneity between the effect sizes of the studies. In fact, an inspection of Figure 3 revealed that the study of Feixas et $\mathrm{a}^{22}$ presented an OR that differed significantly from the mean. The heterogeneity level decreased significantly $\left(I^{2}=33.23\right)$ when this study was removed.

We found similar results when examining group differences with regard to level of conflicts. The mean effect size for this comparison was medium $(d=0.61 ; 95 \% \mathrm{CI}$ : $0.49-0.72)$. With the exception of Compañ et $\mathrm{al}^{24}(d=0.25$; 95\% CI: $-0.26-0.75$ ), all the studies showed that clinical participants had considerably higher individual frequency of IDs than controls (Figure 4). Finally, with regard to the

\begin{tabular}{|c|c|c|c|}
\hline Study name & $\begin{array}{l}\text { Std diff } \\
\text { in means }\end{array}$ & $\begin{array}{l}\text { Lower } \\
\text { limit }\end{array}$ & $\begin{array}{l}\text { Upper } \\
\text { limit }\end{array}$ \\
\hline $\begin{array}{l}\text { Benasayag et al, } \\
\text { unpublished data, } 2001\end{array}$ & 0.84 & 0.48 & 1.19 \\
\hline Feixas et $a^{6}$ & 0.54 & 0.37 & 0.70 \\
\hline Feixas et $a^{22}$ & 0.61 & 0.11 & 1.11 \\
\hline Feixas et al ${ }^{44}$ & 0.40 & 0.13 & 0.68 \\
\hline Feixas et $a^{23}$ & 0.63 & 0.38 & 0.88 \\
\hline Melis et $\mathrm{al}^{27}$ & 0.57 & 0.14 & 1.01 \\
\hline Montesano et $\mathrm{al}^{25}$ & 0.89 & 0.58 & 1.20 \\
\hline Compañ et $\mathrm{al}^{24}$ & 0.25 & -0.26 & 0.75 \\
\hline Soldevilla et al ${ }^{20}$ & 0.73 & 0.28 & 1.19 \\
\hline Random & 0.61 & 0.49 & 0.72 \\
\hline
\end{tabular}




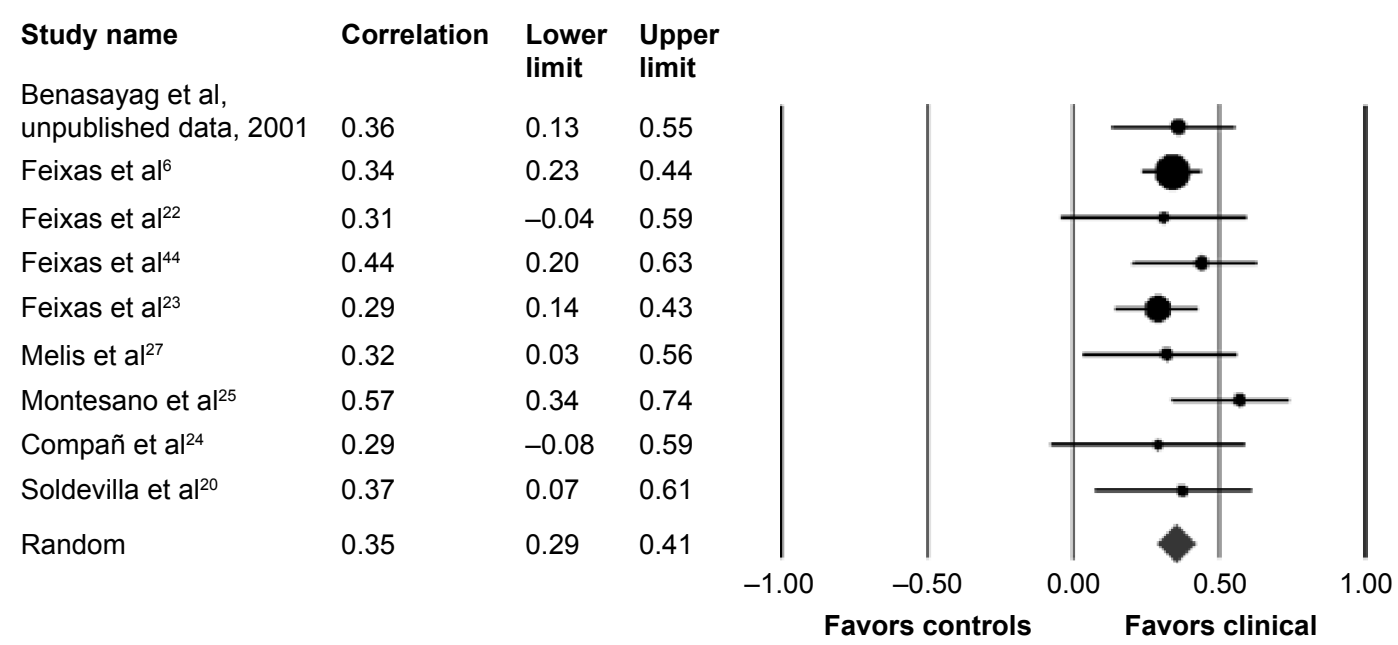

Figure 5 Forest plot for meta-analysis of the relationship between level of conflicts and symptom severity.

question of the relationship between level of conflicts and symptom severity, we also found a medium effect size ( $r=0.35$; 95\% CI: $0.287-0.413)$ indicating a clear association within the clinical samples. Figures $3-5$ show forest plots for these analyses with the relative weights assigned to the studies.

\section{Publication bias}

Although we searched for, and finally included, one manuscript (Benasayag et al, unpublished data, 2001), we carried out a study of publication bias to test whether a bias favoring significant results could influence the effect sizes analyzed. Two different complementary analyses were performed on the global effect size, which was the result of combining all of the outcomes. First, the fail-safe $\mathrm{N}$ index ${ }^{17}$ indicated that, to cancel the global mean effect size obtained, there would have to be 221 unpublished studies with null effects. This index also exceeded Rosenthal's ${ }^{29}$ tolerance level when obtained for each outcome measure (prevalence of IDs: $N=271$; level of conflicts: $\mathrm{N}=288$; symptom severity: $\mathrm{N}=199$ studies). Second, Egger's test for funnel plot asymmetry was non-statistically significant (intercept $=0.178$; $t(7)=1.837, P=0.108)$. Thus, all these results enable us to discard publication bias as a threat to the validity of the estimated effect sizes.

\section{Discussion}

In this article, we presented the results of a meta-analytical review on the role of IDs across a variety of clinical samples. With this purpose, 27 outcomes from nine carefully selected studies were analyzed. The results indicated that IDs play a clinically relevant role in Axis I psychological disorders such as major depression, dysthymia, anxiety, and bulimia, as well as in other clinical conditions. Although the analyses consisted of dissimilar samples, we found a low heterogeneity level among the effect sizes of the studies, indicating that IDs play a similar role across the targeted samples. In light of these results, under a random effects approach, we concluded that there was a substantial difference between clinical conditions compared with controls, with regard to the presence and level of intrapersonal conflict. IDs appear to be significantly more prevalent in clinical samples, regardless of the type of diagnosis. Thus, these results constitute strong evidence for the clinical relevance and transdiagnostic nature of IDs.

It is worth noting, however, that the inclusion of studies that used the same assessment procedure derived from a multicenter research project definitely contributed to the observed homogeneity. Future research could focus on reviewing the role of intrapersonal conflict using different procedures of measurement, such as unbalanced triads, ${ }^{30}$ goal conflicts, ${ }^{31}$ or psychodynamically oriented methods. ${ }^{4}$ This line of research could enhance the development of evidence-based protocols that would assist the therapist when dealing with intrapersonal conflict in psychotherapy. As yet, there are few studies that carry out these approaches with clinical samples while also reaching a satisfactory level of methodological quality.

Our review focused exclusively on clinical conditions, discarding studies based on nonclinical participants even if they controlled for the level of symptoms, ${ }^{32}$ functioning or well-being. ${ }^{33}$ Therefore, results from our review have ecological validity for clinical settings inasmuch as they describe the types of conflicts that lead to human suffering and ambivalence toward change. IDs are particularly ecological because they are not defined a priori by the 
researcher or the clinician, but are rather derived from the clients' subjective manner of constructing their experience. These might be crucial in both psychological and health problems. However, we included only two studies regarding health problems and so results should be interpreted very cautiously for these clinical conditions. On the other hand, the two health conditions included are considered functional disorders. Undoubtedly, the extent to which IDs are relevant for health conditions must be examined more thoroughly in future research.

As expected, a higher level of conflict was statistically associated with symptom severity within targeted samples. The mean effect size indicated a medium correlational strength with a very low level of heterogeneity between samples. Actually, from our theoretical perspective, we cannot assume a large linear relationship between the two variables because we do not equate symptoms with conflicts. Nevertheless, the construction of symptoms and particularly their endurance might be underpinned by conflictual configurations within the self-system. For instance, Michalak et $\mathrm{al}^{2}$ found that the greater the interference the clients' symptomatic behavior presented, the more difficult it was for them to reach their desired goals, whereas a reduction of positive symptom implications led to a better treatment outcome. ${ }^{9}$ Our results can be understood as a confirmation of these clinical observations as we found a significant correlation between the level of conflicts and the severity of the disorder. Nevertheless, we cannot overlook the fact that the available data for the inspection of the relationship between conflict and severity was limited to the symptom level, while disregarding other important factors such as well-being, quality of life, global functioning, number of episodes, duration, and the course of the disorder.

A major concern for the clinical interpretation of our results is the retrospective and cross-sectional nature of the included studies, which precluded causal analysis of reported associations. Longitudinal future research should focus on the influence of IDs over the clinical course, examining its relationship with treatment outcomes, as well as the rates of relapse and recurrence within randomized clinical trials.

Despite the aforementioned limitations, our results lead us to some implications for clinical practice. Findings from our meta-analysis suggest that there is a far from negligible proportion of clients whose cognitive systems are in conflict, and for whom facing changes may pose a dilemma because of invalidation of core aspects of their identity. Moreover, these conflicts are, to a great extent, related to the severity of their symptoms. Therefore, psychotherapists could benefit considerably from screening for the presence of IDs in their patients insofar as it could enhance case formulation and shed light on the clients factors that maintain or worsen the symptoms related to the disorder. This should be a priority with patients experiencing difficulties to overcome their symptoms.

Indeed, IDs can be a focus of therapeutic work and can help in personalizing the design of a treatment to fit a client's subjective experience. Recently, a dilemma-focused intervention protocol has been manualized for depression in combination with a cognitive behavioral group intervention. ${ }^{34}$ However, this "add on" intervention could be applied to different diagnostic entities and in combination with a variety of approaches in psychotherapy.

There are already some approaches devised for working with inner conflict and ambivalence. ${ }^{35-39}$ Perhaps the most widespread is the motivational interviewing approach, ${ }^{37}$ which has investigated the role of motivational interventions for engaging and encouraging change in a range of psychological problems. ${ }^{40}$ This model shares some features with the dilemma-focused intervention, for instance, both emphasize the patients' responsibility in taking decisions about their future behavior; they use techniques to explore decisional processes and both understand resistance as a sign indicating that the therapists are not correctly attuning their interventions to the state of the patient. There are, however, some differences between the approaches. As described by Feixas and Compañ, ${ }^{41}$ the main discrepancy between both approaches is the way in which ambivalence is conceived, as well as its relationship with symptom maintenance. For the motivational interviewing approach, ambivalence emerges whenever there is an attachment to an addictive/problematic behavior. In brief, this attachment can be caused by learning processes and conditioning, or the utilization of the addictive/ problematic behavior as a coping mechanism to face difficult or unpleasant emotional states (eg, overcome inhibition). In the dilemma-focused approach, the difficulty to change is explained in terms of the association of the symptom with the patient's sense of identity. That is, when the symptom is associated with a core personal value, changing would imply stop being oneself in such core aspect of the self. So, the ambivalence is not explained only by the learning processes, but also by the fact that it has personal meanings that serve as a protective function against invalidation of core aspects of identity.

Therefore, therapists using the dilemma-focused protocol shall reconcile clients' motivation to change with core aspects of their identity. In so doing, therapists' efforts should 
be directed toward helping clients to achieve their desired changes (as reflected in discrepant constructs of IDs), but without disregarding the likely positive implications (usually implicit) that symptoms could have within the clients' cognitive system (as reflected in congruent constructs of IDs). Future longitudinal research should elucidate whether targeting inner conflicts in the therapy process may benefit therapeutic adherence and the maintenance of change in the long run.

There are some specific clinical situations in which the dilemma-focused intervention within a broader psychological treatment might be also helpful. For instance, some patients with a personality disorder (eg, borderline) may criticize themselves for failing to overcome their symptoms and, in the process, further undermine their recovery. A dilemma-focused approach might lower the level of self-criticism by providing the understanding of the complexity of goal accomplishment and revealing other competing but legitimate goals, thus creating a difficult dilemma for the person. In this sense, the patient understanding that there are reasons (although non-apparent) for not pursuing his or her goals might enlarge his or her view of the problem and promote self-acceptance. Future research might examine the prevalence of conflicts in Axis II disorders and compare to the indexes found on this review. Tentatively, it could be hypothesized that IDs could be more prevalent in borderline patients given that they often present certain level of dissociation and identity instability.

In some other clinical situations, IDs might reflect what has been known in the clinical literature as "secondary gains" and, thus, become an alternative method to gauge these well-known conflicts in a systematic way. In fact, the body of research on secondary gains is not very extensive mainly due to the difficulty of the notion to be operationalized in order to be included in research studies. In contrast, the series of studies reviewed in this article show that the notion of ID is notion amenable to be researched and to provide consistent results which, in turn, can be regarded as an indirect support for the "secondary gain" traditional notion.

It is true that the difficulty to tap internal conflicts has limited the body of available research on this longstanding topic. Broadly, there are two clusters of methods to investigate the role of interpersonal conflicts. On the one hand, those methods using explicit measures of conflict in which the patient is aware of the conflict, yielded interesting results when examining interference between personal goals. ${ }^{1}$ However, these methods may be more sensitive to social desirability and self-presentation effects, which may limit their applicability in clinical settings. On the other hand, specific methods for measuring implicit conflicts have been devised from a range of psychological approaches using observational coding systems ${ }^{4}$ or computerized methodologies. ${ }^{42,43}$ A common critique of these methods is that the elements involved in the assessment are defined in advance by the researcher. Nevertheless, these last methods have demonstrated a greater potential for understanding the influence of conflicts and cognitions in behavior and psychopathology.

The IDs approach could be classified within this second category but with the advantage that the elements involved in the detected conflict are not defined by the researcher or therapist, but they stem from the patients' personal construct repertory. Further research might study commonalities and differences between the IDs' approach and other computerized methods for assessing implicit cognitive processes in order to ascertain the psychological mechanisms involved in each one and explore the clinical benefits of integrating both in the assessment of patients.

\section{Acknowledgment}

The authors thank Terry Georgiou for his help in the English editing of this paper.

\section{Disclosure}

The authors report no conflicts of interest in this work.

\section{References}

1. Emmons RA, King LA. Conflict among personal strivings: immediate and long-term implications for psychological and physical well-being. J Pers Soc Psychol. 1988;54(6):1040-1048.

2. Michalak J, Heidenreich T, Hoyer J. Goal conflicts and goal integration: theory, assessment, and clinical implications. In: Cox WM, Klinger E, editors. Handbook of Motivational Counseling: Goal-Based Approaches to Assessment and Intervention with Addiction and Other Problems. 2nd ed. London: Wiley; 2011:89-107.

3. Grosse Holtforth M, Grawe K. Der Inkongruenzfragebogen (INK) - Ein Messinstrument zur Analyse motivationaler Inkongruenz [The incongruence questionnaire (INK) - An instrument for analyzing motivational incongruence]. Zeitschrift für Klinische Psychologie und Psychotherapie 2003;32(4):315-323. German.

4. Cierpka M, Grande T, Rudolf G, von der Tann M, Staschf M. The operationalised psychodynamic diagnostic system: clinical relevance, reliability and validity. Psychopathology. 2007;40(4):209-220.

5. Arnow BA, Manber R, Blasey C, et al. Therapeutic reactance as a predictor of outcome in the treatment of chronic depression. J Consult Clin Psychol. 2003;71(6):1025-1035.

6. Feixas G, Saúl LA, Ávila-Espada A. Viewing cognitive conflicts as dilemmas: implications for mental health. J Constr Psychol. 2009;22(2): $141-169$

7. Feixas G, Saúl LA. The Multi-Center Dilemma Project: an investigation on the role of cognitive conflicts in health. Span J Psychol. 2004, 7(1):69-78.

8. Kelly GA. The Psychology of Personal Constructs. New York, NY: Routledge; 1955/1991. 
9. Feixas G, Saúl LA, Winter D, Watson S. Un estudio naturalista sobre el cambio de los conflictos cognitivos durante la psicoterapia [A naturalistic study about change in cognitive conflicts during psychotherapy]. Apuntes de Psicología. 2008;26(2):243-255. Spanish.

10. Botella J, Gambara H. Doing and reporting a meta-analysis. Int J Clin Health Psychol. 2006;6:425-440.

11. Sánchez-Meca J, Marín-Martínez F, López-López JA. Meta-análisis e intervención psicosocial basada en la evidencia [Meta-analysis and evidence-based psychosocial intervention]. Psychosocial Intervention. 2011;20:95-107. Spanish.

12. Wells G, Shea B, O'Connell D, et al. The Newcastle-Ottawa Scale (NOS) for assessing the quality of nonrandomized studies in meta-analyses. Ottawa, Canada: Ottawa Hospital Research Institute; 2012. Available from: http://www.ohri.ca/programs/clinical_epidemiology/oxford. asp. Accessed February 6, 2015.

13. Feixas G, Cornejo JM. Correspondence Analysis for Grid Data and Reperory Grid Manual [Computer Software]. Barcelona: Psimedia; 2002.

14. Cohen J. Statistical Power Analysis for the Behavioral Sciences. 2nd ed. Hillsdale, NJ: Lawrence Erlbaum Associates; 1988.

15. Field AP, Gillett R. How to do a meta-analysis. Br J Math Stat Psychol. 2010;63:665-694.

16. Lipsey MW, Wilson DB. Practical Meta-analysis. Thousand Oaks, CA: Sage; 2001.

17. Rothstein HR, Sutton AJ, Borenstein M. Publication Bias in Metaanalysis: Prevention, Assessment, and Adjustments. Chichester: Wiley; 2005.

18. Borenstein M, Hedges L, Higgins J, Rothstein H. Comprehensive Meta-analysis Version 2. Englewood, NJ: Biostat Inc.; 2005.

19. American Psychiatric Association. Diagnostic and Statistical Manual of Mental Disorders. 4th ed, text rev. Washington, DC: Author; 2000.

20. Soldevilla JM, Feixas G, Varlotta N, Cirici R. Characteristics of the construct system of women victims of intimate partner violence. $J$ Constr Psychol. 2014;27(2):105-119.

21. Derogatis LR. Symptom Checklist 90 Revised. Minneapolis, MN: NCS Pearson; 1994.

22. Feixas G, Montebruno C, Dada G, Del Castillo M, Compañ V. Self construction, cognitive conflicts and polarization in bulimia nervosa. Int J Clin Health Psychol. 2010;10(3):445-457.

23. Feixas G, Montesano A, Compañ V, et al. Cognitive conflicts in major depression: between desired change and personal coherence. Br J Clin Psychol. 2014;53(4):369-385.

24. Compañ V, Feixas G, Varlotta-Domínguez N, et al. Cognitive factors in fibromyalgia: the role of self-concept and identity related conflicts. J Constr Psychol. 2011;24(1):56-77.

25. Montesano A, Feixas G, Erazo-Caicedo MI, Saúl LA, Dada G, Winter D. Cognitive conflicts and symptom severity in dysthymia: "I'd rather be good than happy." Salud Mental. 2014;37(1):41-48.

26. Beck AT, Brown G, Steer RA. Beck Depression Inventory II Manual. 2nd ed. San Antonio, TX: The Psychological Corporation; 1996.

27. Melis F, Feixas G, Varlotta N, et al. Conflictos cognitivos (dilemas) en pacientes diagnosticados con trastornos de ansiedad [Cognitive conflicts (dilemmas) in patients diagnosed with anxiety disorders]. Revista Argentina de Clinica Psicologica. 2011;20(1):41-48. Spanish.
28. Spielberger CD. State-Trait Anxiety Inventory: Bibliography. 2nd ed. Palo Alto, CA: Consulting Psychologists Press; 1989.

29. Rosenthal R. The "file drawer problem" and tolerance for null results. Psychol Bull. 1979;86:638-641.

30. Lauterbach W. The measurement of personal conflict. Psychother Res. 1996;6:213-225.

31. Riediger M. Interference and facilitation among personal goals: age difference and associations with well-being and behavior. In: Little BR, Salmera-Aro K, Philips SD, editors. Personal Project Pursuit: Goals, Action and Human Flourishing. Mahwah, NJ: Lawrence Erlbaum Associates; 2007:119-143.

32. Dada G, Feixas G, Compañ V, Montesano A. Self-construction, cognitive conflicts, and disordered eating attitudes in young women. $J$ Constr Psychol. 2012;25(1):70-89.

33. Badzinski SI, Anderson JA. An exploration of the identification of implicative dilemmas and their relationship to personal construct theory-congruent measures of psychological well-being in nonclinical samples. J Constr Psychol. 2012;25(1):1-33.

34. Feixas G, Bados A, García-Grau E, et al. Efficacy of a dilemma-focused intervention for unipolar depression: study protocol for a multicenter randomized controlled trial. Trials. 2013;14:144.

35. Ecker B, Ticic R, Hulley L. Unlocking the Emotional Brain: Eliminating Symptoms at Their Roots Using Memory Reconsolidation. New York, NY: Routledge; 2012.

36. Mansell W, Carey TA, Tai SJ. A Transdiagnostic Approach to CBT Using Method of Levels Therapy: Distinctive Features. London: Routledge; 2012.

37. Miller WR, Rollnick S. Motivational Interviewing: Helping People Change. 3rd ed. New York, NY: Guilford Press; 2013.

38. Ribeiro AP, Mendes I, Stiles WB, Angus L, Sousa I, Gonçalves MM. Ambivalence in emotion-focused therapy for depression: the maintenance of problematically dominant self-narratives. Psychother Res. 2014;24:702-710

39. Sutherland O, Peräkylä A, Elliott R. Conversation analysis of the two-chair self-soothing task in emotion-focused therapy. Psychother Res. 2014;24(6):738-751.

40. Arkowitz H, Westra HA, Millar W, Rollnick S. Motivational Interviewing in the Treatment of Psychological Problems. New York, NY: Guilford; 2008.

41. Feixas G, Compañ V. Manual de intervención centrada en dilemas para la depresión [Handbook of dilemma-focused intervention for depression]. Bilbao: Desclée DeBrouwer; 2015. Spanish.

42. Lauterbach W, Newman CF. Computerized intrapersonal conflict assessment in cognitive therapy. Clin Psychol Psychother. 1999;6: $1-18$.

43. Gemar MC, Segal ZV, Sagrati S, Kennedy SJ. Mood-induced changes on the Implicit Association Test in recovered depressed patients. J Abnorm Psychol. 2001;110(2):282-289.

44. Feixas G, Montesano A, Erazo-Caicedo MI, Compañ V, Pucurull O. Implicative dilemmas and symptom severity in depression: a preliminary and content analysis study. J Constr Psychol. 2014;27(2):31-40.
Neuropsychiatric Disease and Treatment

\section{Publish your work in this journal}

Neuropsychiatric Disease and Treatment is an international, peerreviewed journal of clinical therapeutics and pharmacology focusing on concise rapid reporting of clinical or pre-clinical studies on a range of neuropsychiatric and neurological disorders. This journal is indexed on PubMed Central, the 'PsycINFO' database and CAS,

\section{Dovepress}

and is the official journal of The International Neuropsychiatric Association (INA). The manuscript management system is completely online and includes a very quick and fair peer-review system, which is all easy to use. Visit http://www.dovepress.com/testimonials.php to read real quotes from published authors. 